the right side, immediately above Poupart's ligament.

When admitted, the abdomen was very tender, and an obscure tumour could be felt on the right side of the linea alba above the groin; it was very firm, and pressure upon it caused pain. His bowels were moved daily, the fæces being generally light coloured and loose; the swelling continued to extend upwards until the 23rd of July, when it burst at the umbilicus, and a considerable quantity of fæculent matter of a light colour and thin consistence was discharged; this gave immediate relief to the pain which had accompanied its formation. Fæces were discharged almost daily from the umbilicus, as well as from the anus, the quantity varying in proportion to the violence of the diarrhoea which was present. He died about the middle of September, and on examination the following appearances were detected:-

The lower portion of the ileum and the cæcum were extensively thickened in consequence of lymph having been thrown out on some previous occasion. A sinus was found extending from the cæcum to the umbilicus; it was formed by the appendix vermiformis cæci, which was bent upwards and adherent to the anterior parietes of the abdomen, to within about an inch of the umbilicus, with which it communicated by a short fistulous canal. The appendix was thickened and dilated, and the shell of a hazel nut was impacted in its middle portion.

\section{CARIES OF THE STERNUM WITH} ULCERATION OF THE LUNG.

Sir:-As the following case may present something of interest to many of your readers, I shall feel obliged by its insertion in your valuable Journal. I am Sir, your obedient servant,

Tiverton, June 22, 1840.

\section{G. Coward.}

ULCERATION OF TIE LUNG, ORIGINATING IN CARIES OE THE STERNUM.

A full, powerful man, but of a decidedly strumous diathesis, was affected with circumscribed inflammation over the sternum, and oppression in the chest, about three months since; symptoms which he attributed to an accidental chill. The local appearances at this time were circumscribed odematous tumefaction; heat, pain, and redness, situated opposite the mamma a little to the right of the mesial line. The patient complained of deep-seated pain and oppression beneath the externally affected part; there was congh, dyspnœa, and slight expectolation. The general symptoms were febrile : quick, hard pulse; hot skin, thirst, white tongue, and constipated bowels. As the morbid process proceeded, the ex istence of pus became evident, and the swelling increased, with fluctuation, whilst the geveral symptoms assumed the hectic type. By the application of poultices the progress of the pus to the surface was facilitated, and after some days the abscess spontaneously broke, discharging ill-conditioned and offensive matter. The sternum was found to be carious : it was rough and excavated, and continued to discharge offensive pus as the morbid process increased. Notwithstanding active and judicious treatment, the disease continued to exlend until the sternum was completely perforated and the lung exposed, which had doubtless contracted extensive adhesions by the opposing surfaces of the pleuræe during the increase of disease. The lung itself now became affected: a large ulcerated cavity was soon formed (most probably in the middle lobe of the right side), and air escaped easily by any respiratory effort. In this condition, with but slight aggravation of symptoms, he still continues, and at present he presents the following morbid signs, local and general. There is an ulcerating cavity at the point indicated, of about two or three inches in extent, through which air readily escapes in any powerful respiratory effort, but not during ordinary breathing; the margin of this cavity is red and tumid, and there is considerable tumefaction over a similar spot on the left side. The patient complains very little of pain, but the inflamed portions are extremely tender. There is occasional cough and expectoration, and the fever present is hectic: there is a permanently quick pulse, exacerbations of fever occurring in remittent paroxysms, occasional night sweats and thirst, whilst the strength fails rapidly; but there is no emaciation, as of late; the countenance has become puffy and odematous, and of a leucophlegmatic aspect; and there is a decided tendency to general anasarcous effusion, as is evinced in the appearance and feel of the general surface.

Judging from the negative effect of treatment, the strumous diathesis, and the steady but decided advance of bad symptoms, the prognosis is unfavourable; whilst, on the contrary, the patient's youth, usual strength, and active habits, are in his favour.

Health of the ARmy.-In the Supple. mentary Estimate of the Office of Ordnance, 1840-1841, laid, on the 1st instant, before the House of Commons, the sum of f27S1 is proposed to be expended in the erection of Ball and Fives Courts in the Barracks of Great Britain, under the au. thority of a Treasury resolution, passed in April last. 